\title{
The impact of thiopurine-S-methyltransferase genotype on the adverse drug reactions to azathioprine in patients with inflammatory bowel diseases
}

\author{
Hlavaty $\mathrm{T}^{1}$, Batovsky $\mathrm{M}^{2}$, Balakova $\mathrm{D}^{3}$, Pav $\mathrm{I}^{2}$, Celec $\mathrm{P}^{3,4}$, Gregus $\mathrm{M}^{6}$, Zakuciova $\mathrm{M}^{7}$, Hlista $\mathrm{M}^{8}$, \\ Horakova $\mathrm{M}^{9}$, Desatova $\mathrm{B}^{2}$, Koller $\mathrm{T}^{1}$, Toth $\mathrm{J}^{1,10}$, Kadasi $\mathrm{L}^{3,5}$, Huorka $\mathrm{M}^{1}$ \\ Department of Internal Medicine V, Division of Gastroenterology and Hepatology, University Hospital \\ Bratislava Ruzinov, Slovakia. tibor.hlavaty2@gmail.com
}

\begin{abstract}
Background and aims: The thiopurine drugs, azathioprine (AZA) and 6-mercaptopurine, are established in the treatment of inflammatory bowel diseases (IBD). Polymorphisms in thiopurine S-methyltransferase (TPMT) gene have been associated with adverse drug reactions (ADRs) to AZA.

Methods: The aim of this study was to evaluate TPMT polymorphisms and AZA-related toxicity in a Slovak cohort of 220 IBD patients treated with AZA. In every patient, the dose and duration of AZA therapy, concomitant 5-aminosalicylate (5-ASA) medication, frequency, type, time to onset, dose of ADR and concomitant 5-ASA at the onset of ADR were recorded. Each patient was also genotyped for the presence of variant TPMT alleles $\left({ }^{*} 2,{ }^{*} 3 A,{ }^{*} 3 B,{ }^{*} 3 C\right)$. Frequency, type and circumstances of ADRs were compared according to TPMT status. Results: Of the 220 patients, $205(93.2 \%)$ were wild-type (TPMT*1/*1), one $(0.5 \%)$ carried a TPMT*1/*3C allele, $13(5.9 \%)$ carried TPMT * $1 /{ }^{*} 3 \mathrm{~A}$ allele and one was homozygous for TMPT *3A allele. No TPMT *2 mutation was found. The incidence of adverse drug reactions was 62/205 (30.2\%) in the wild-type group as compared to $13 / 15(86.7 \%)$ in the TPMT mutation group, $p=2.10^{-5}$. Leukopenia (WBC $<3.0^{*} 10^{\wedge}$ 9/L) occurred in $21 / 205$ (10.2 \%) patients with wild type TPMT versus $11 / 15$ (73.3 \%) patients with TPMT mutations, $p=0.000001$. There was no significant difference between TMPT groups in gastrointestinal or other ADRs. No impact of 5-ASA on the incidence and severity of AZA adverse drug reactions was observed.

Conclusion: The incidence of leukopenia in TPMT mutant patients was significantly higher and more severe as compared to TPMT wild type patients. We observed no impact of concomitant 5-ASA therapy on AZA induced toxicity (Tab. 4, Fig. 2, Ref. 37). Full Text in PDF www.elis.sk.

Key words: inflammatory bowel diseases, Crohn's disease, ulcerative colitis, therapy, azathioprine, 6-mercaptopurine, thiopurine-S-methyltransferase, polymorphisms, adverse drug reaction.
\end{abstract}

\begin{abstract}
Abbreviations used: 5-ASA-5-aminosalicylic acid, 6-MP-6-mercaptopurine, ADR - adverse drug reaction, AZA - azathioprine,

The Slovak IBD study group (SK IBD)

${ }^{1}$ Department of Internal Medicine V, Division of Gastroenterology and Hepatology, University Hospital Bratislava Ruzinov, Slovakia, ${ }^{2}$ Department of Gastroenterology, University Hospital Bratislava Petrzalka, Slovakia, ${ }^{3}$ Slovakia Department of Molecular Biology, Comenius University, Bratislava, Slovakia, ${ }^{4}$ Institute of Molecular Biomedicine, Comenius University, Bratislava, Slovakia, ${ }^{5}$ Institute of Molecular Physiology and Genetics, Slovak Academy of Sciences, Bratislava, ${ }^{6} \mathrm{KM}$ Gastroenterology centre Nitra, Slovakia, ${ }^{7}$ Department of Internal Medicine I, Division of Gastroenterology and Hepatology, University Hospital Kosice, Slovakia, ${ }^{8}$ Department of Internal Medicine, Hospital Trencin, Slovakia, ${ }^{9}$ Department of Internal Medicine II, Division of Gastroenterology and Hepatology, University Hospital Martin, Slovakia, and ${ }^{10}$ Medissimo Health Care Centre, Division of Gastroenterology, Bratislava, Slovakia
\end{abstract}

Address for correspondence: T. Hlavaty, MD, PhD, Department of Internal Medicine, Division of Gastroenterology and Hepatology, University Hospital Bratislava, Ruzinovska 6, SK-826 06 Bratislava, Slovakia. Phone/Fax: +421.2.48234905

Acknowledgement and support: This work was supported by a scientific grant VEGA No 1/007/08 from the Scientific Grant Agency (VEGA) of the Ministry of Education of the Slovak Republic.
CD - Crohn's disease, IBD - inflammatory bowel disease, TPMT - thiopurine-S-methyltransferase, UC - ulcerative colitis.

Inflammatory bowel diseases (IBD) are a group of chronic relapsing inflammatory diseases comprising Crohn's disease (CD), ulcerative colitis (UC) and indeterminate colitis. The thiopurine drugs azathioprine (AZA) and 6-mercaptopurine (6-MP) have been shown to be an effective therapy for inducing and maintaining remission in both Crohn's disease and ulcerative colitis (1-6). Adverse drug reactions occur in $15-30 \%$ of patients and often require dose reduction or drug discontinuation (7-9). The most often adverse drug reactions are nausea/dyspepsia, bone marrow suppression, hepatic toxicity, pancreatitis and allergic reactions. The most severe side effect, observed in up to $10 \%$ of patients, is the bone marrow suppression with neutropenia and/or pancytopenia, which can be lethal. Generally it is a dose-related adverse drug reaction; however there is a significant inter-individual variability, mostly due to polymorphisms in genes encoding the metabolic pathways of thiopurines.

There are three major metabolic pathways of thiopurines mediated by enzymes hypoxantine phosphoribosyl transferase (HPRT), 
thiopurine-S-methyltransferase (TPMT) and xantine oxidase (XO). The intracellular formation of active substances is mediated by HPRT and results in the formation of thioguanine nucleotides (TGN). They are purine antagonists and exert cytotoxic and immunosuppressive effects partially by direct incorporation of TGN into DNA with subsequent inhibition of DNA, RNA and protein synthesis (10). It has been also shown that TGN induce lymphocyte apoptosis through replacing guanine triphosphate and subsequent suppression of Rac1 activity (11). Selective inhibition of inflammatory genes expression in activated $\mathrm{T}$ lymphocytes is another observed mechanism of action of thiopurines (12).

Methylation and oxidative metabolic pathways mediated by TPMT and XO compete with the HPRT-mediated formation of TGNs. The methylation of 6-MP metabolite thioinosine monophosphate (TIMP) by TPMT leads to the production of methylthiosine monophosphate (meTIMP) which is a potent inhibitor of purine de novo synthesis $(13,14)$. High meTIMP levels are also associated with an increased risk of hepatotoxicity $(15,16)$. There are several known polymorphisms in TPMT gene associated with a decrease in TPMT activity and myelotoxicity due to high TGN metabolite concentrations $(17,18)$.

In our retrospective study we wanted to analyze the association of major TPMT polymorphisms and various adverse drug reactions in a large cohort of IBD patients treated with AZA. Specifically we were interested in the interaction of TPMT genotype with the dose, concomitant 5-ASA medication and time to onset of the adverse drug reactions.

\section{Materials and methods}

\section{Patient cohort}

The patient cohort consisted of 220 consecutive IBD patients who were treated with AZA or 6-MP either currently or in the past. The patients were recruited from six participating IBD centers from the Slovak IBD study group (SK-IBD) between January 1, 2009 and May 31, 2009. All eligible patients who signed the informed consent for the genetic examination of TPMT polymorphisms and agreed to participate in the study were included. No other genetic test has been or will be done on biological material obtained in this study unless explicitly consented to and signed by the patient.

Clinical characteristics of each patient including sex, age, current and previous azathioprine medication, start and duration of AZA therapy, current 5-aminosalycilic acid (5-ASA) therapy and dose, current blood count, CRP and creatinine levels were collected.

\section{Adverse drug reactions to azathioprine therapy}

Medical records of every patient were screened for the history of adverse drug reactions (ADR) to azathioprine therapy. In most patients a detailed history including medical and laboratory data was readily available in his/her physical and/or electronic patient data file. In patients referred to participating centers, the referring physician was contacted and consulted if necessary. In case of an adverse drug reaction, the date of the adverse drug reaction, duration of AZA therapy before the adverse drug reaction, the dose of AZA, concomitant 5-aminosalycilic acid (5-ASA) therapy and its dose, blood count, creatinine and C-reactive protein (CRP) levels were recorded.

Leukopenia was defined as a white blood cells count (WBC) $<3.0 \times 10^{9} / \mathrm{ml}$. Anemia was considered AZA-related if developed quickly during AZA therapy in absence of apparent bleeding, hemoglobine levels decreased below $100 \mathrm{~g} / \mathrm{L}$ and reversed to normal after AZA cessation or dose reduction. Trombocytopenia was defined as platelets count $<100 \times 10^{9} / \mathrm{L}$. Hepatotoxicity was defined as an elevation of alanine transaminase (ALT) or aspartate aminotransferase (AST) levels of at least twice the upper limit of normal. Pancreatitis was defined as an elevation of serum amylase or lipase of more than twice the upper limit of normal, associated with abdominal pain. The diagnosis of allergic reaction, dyspepsia and other adverse drug reactions was made by the clinician, who treated the respective patient.

\section{TPMT polymorphism genotyping}

Total genomic DNA was extracted from peripheral blood cells using the Puregene Blood Core kit (Qiagen, Hilden, Germany). PCR method was used for preamplification of three fragments containing polymorphisms in TPMT gene. The 279-bp fragment containing the polymorphism A719G was amplified with primers A719G-F (5' -CACCCAGCCAATTTTGAGTAT-3') and A719GR (5'-TCCATTACATTTTCAGGCTTTAG-3'). The 398-bp fragment containing the polymorphism G460A was amplified with primers G460A-F (5'-AAACGCAGACGTGAGATCCT-3') and G460A-R (5'-GCCTTACACCCAGGTCTCTG-3'). The 252-bp fragment containing the polymorphism $\mathrm{G} 238 \mathrm{C}$ was amplified with primers G238C-F (5'-GCATGTTCTTTGAAACCCTATGA-3') and G238C-R (5'-GGAATTTCGGTGATTGGTTC-3'). Amplifications were performed in a $10 \mu \mathrm{l}$ reaction volume containing $50 \mathrm{ng}$ of genomic DNA, 1x concentrated 5PRIME HotMasterMix (5PRIME, Hamburg, Germany) and 2.5 pmol of each primer. The amplifications were performed on TC-412 thermocycler (TECHNE, Bibby Scientific, Stone, UK). Samples were denatured at $95{ }^{\circ} \mathrm{C}$ for 4 minutes, followed by 30 cycles at $95^{\circ} \mathrm{C}$ for $40 \mathrm{sec}-$ onds, $58^{\circ} \mathrm{C}$ for 30 seconds, and $72^{\circ} \mathrm{C}$ for 40 seconds. Amplification cycles were followed by a ten-minute final extension at 72 ${ }^{\circ} \mathrm{C}$. These fragments were then used as a template for sequencing reactions. For sequence analysis of samples, BigDye Terminator v3.1 Cycle Sequencing Kit (Applied Biosystems, Carlsbad, CA, USA) and the ABI PRISM® 3100 Genetic Analyzer (Applied Biosystems, Carlsbad, CA, USA) instrument was used. Obtained sequences were analyzed by Sequencing Analysis Software v 5.4 (Applied Biosystems, Carlsbad, CA, USA) and Chromas Lite v 2.01 (Technelysium Pty Ltd, Australia) and subsequently compared to GenBank reference sequences using BLAST sequence alignment software (http://blast.ncbi.nlm.nih.gov/genbank).

\section{Statistical analysis}

All statistical analyses were performed using SPSS 18.0 statistical software package (IBM SPSS Inc., Chicago, IL, USA). Nominal and ordinal variables (sex, current AZA use, genotype, adverse drug reactions) were analyzed with Chi square test with 
Tab. 1. Clinical characteristics of the cohort.

\begin{tabular}{|c|c|c|c|c|}
\hline \multirow[t]{2}{*}{ Characteristics } & \multirow{2}{*}{$\begin{array}{l}\text { Whole cohort } \\
\qquad(\mathrm{N}=220)\end{array}$} & \multicolumn{2}{|c|}{ AZA use } & \multirow[t]{2}{*}{$\mathrm{p}$ value } \\
\hline & & $\begin{array}{l}\text { Current } \\
(n=169)\end{array}$ & $\begin{array}{c}\text { Past } \\
(\mathrm{n}=51)\end{array}$ & \\
\hline \multicolumn{5}{|l|}{ Clinical Characteristics } \\
\hline Sex (Male/Female) & $119 / 101(54 / 46 \%)$ & $100 / 69(59 / 41 \%)$ & $19 / 32(37 / 63 \%)$ & 0.006 \\
\hline Age $($ Mean \pm SD) & $37.1 \pm 12.4$ & $36.3 \pm 12.3$ & $39.7 \pm 12.3$ & n.s. \\
\hline Age at diagnosis $($ Mean $\pm \mathrm{SD})$ & $30.2 \pm 12.2$ & $30 \pm 11.7$ & $30.8 \pm 13.5$ & n.s \\
\hline Disease duration (Mean \pm SD) & $6.9 \pm 6.0$ & $6.3 \pm 4.9$ & $8.9 \pm 8.4$ & 0.007 \\
\hline \multicolumn{5}{|l|}{ AZA medication } \\
\hline AZA induction (years after Dx) (Mean \pm SD) & $3.8 \pm 5.5$ & $3.2 \pm 4.5$ & $5.8 \pm 7.9$ & 0.005 \\
\hline Duration of AZA therapy (years) (Mean \pm SD) & $3.3 \pm 3.0$ & $3.3 \pm 3.0$ & $4.9 \pm 3.4$ & n.s \\
\hline Concomitant 5-ASA & $147(66.8 \%)$ & $112(66.3 \%)$ & $35(68.6 \%)$ & n.s. \\
\hline \multicolumn{5}{|l|}{ Laboratory results } \\
\hline WBC (Mean \pm SD) & $7.2 \pm 2.9$ & $7.1 \pm 2.8$ & $7.8 \pm 3.1$ & n.s. \\
\hline Lymphocyte count (No of patients/Mean \pm SD) & $142 / 1.5 \pm 3.2$ & $110 / 1.5 \pm 3.2$ & $32 / 1.6 \pm 2.9$ & n.s. \\
\hline $\operatorname{MCV}($ Mean \pm SD $)$ & $89.8 \pm 7.5$ & $89.9 \pm 7.3$ & $89.2 \pm 8.2$ & n.s \\
\hline
\end{tabular}

Yates correction. If any cell of the contingency table contained a value of less than 5, Fisher exact test was used instead. For each statistically positive result, an odds ratio (OR) with $95 \%$ confidence intervals (CI) was calculated. Continuous variables (age, duration of AZA use, laboratory results) were first analyzed for normality with Kolmogorow-Smirnoff test and then tested by the Student t-test or ANOVA techniques as appropriate. The distribution of alleles was tested with equilibrium analysis. $\mathrm{P}$ value $<0.05$ was considered statistically significant.

\section{Results}

The cohort consisted of 220 patients. All used azathioprine; there was no 6-MP use among the patients in our cohort. The clinical characteristics of the cohort as well as the comparison between the current and past AZA users are given in Table 1.

All patients were successfully genotyped for the studied TPMT polymorphisms. A total of $205(93.2 \%)$ patients carried a normal TPMT $* 1 / * 1$ genotype. There was no patient with TPMT $* 2$ allele. In 15 patients $(6.8 \%)$ a TPMT $* 3$ mutation was diagnosed. There were 13 patients $(5.9 \%)$ with TPMT * $1 / * 3$ A heterozygote genotype, 1 patient $(0.4 \%)$ with TPMT $* 1 / * 3 \mathrm{C}$ heterozygote genotype, and one patient homozygous for TPMT *3A genotype $(0.4 \%)$.

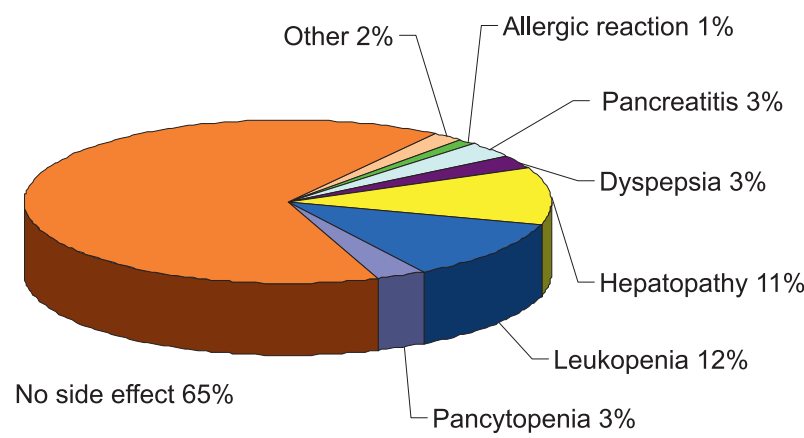

Fig. 1. The frequency of various adverse drug reactions to azathioprine in the study cohort.

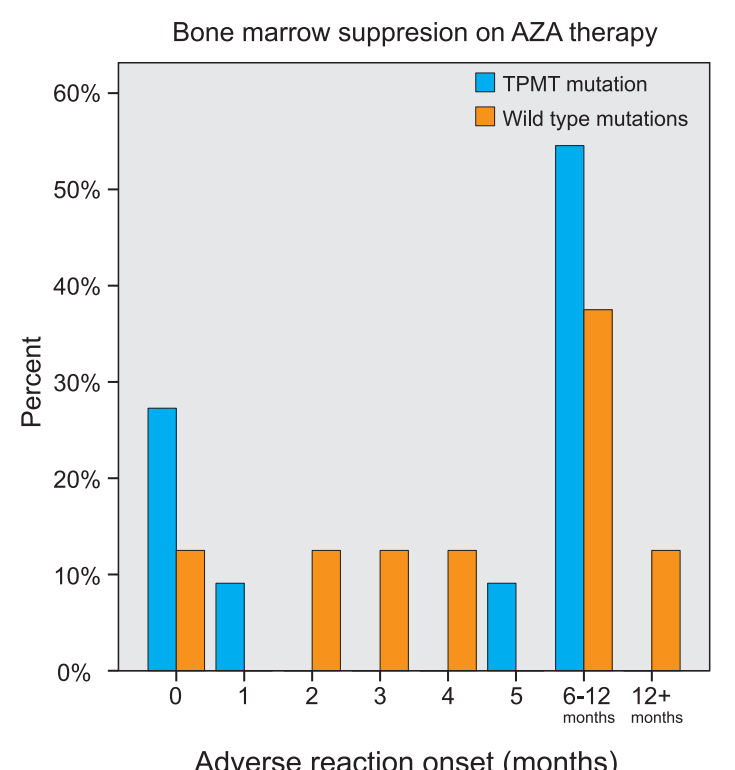

Fig. 2. Time to onset of bone marrow suppression according to TPMT genotype in IBD patients treated with azathioprine.

Adverse drug reactions to azathioprine therapy

A total of 75 (34.1\%) patients experienced some type of adverse drug reactions during the course of azathioprine therapy. The most frequent adverse drug reactions were myelotoxicity (in 32 ; $14.5 \%$ ) and hepatotoxicity (in $24 ; 10.9 \%$ ) of patients. The overview of frequency of adverse drug reaction is given in Figure 1. In 43 patients, the therapy has to be discontinued due to adverse drug reactions while in 32 patients, the adverse drug reactions resolved over time or after the decrease in AZA dose. Adverse drug reactions in current and past AZA users are given in Table 2.

Adverse drug reactions to azathioprine therapy according to TPMT genotype

In the next step we analyzed the influence of TPMT genotype on the incidence of adverse drug reactions. Patients with any TPMT *3 
Tab. 2. Adverse drug reactions to azathioprine therapy in our cohort, in past and current AZA users.

\begin{tabular}{|c|c|c|c|}
\hline \multirow[t]{2}{*}{ Adverse drug reaction type } & \multicolumn{2}{|c|}{ AZA therapy } & \multirow{2}{*}{$\begin{array}{c}\text { Cohort } \\
\text { total } \\
(\mathrm{n}=220)\end{array}$} \\
\hline & $\begin{array}{l}\text { Current } \\
(\mathrm{n}=169)\end{array}$ & $\begin{array}{c}\text { Past } \\
(\mathrm{n}=51)\end{array}$ & \\
\hline Myelotoxicity & $16(9.5 \%)$ & $16(31.4 \%)$ & $32(14.5 \%)$ \\
\hline Leukopenia & $15(8.9 \%)$ & $11(21.6 \%)$ & $26(11.8 \%)$ \\
\hline Bi- or Pancytopenia & $1(0.6 \%)$ & $5(9.8 \%)$ & $6(2.7 \%)$ \\
\hline Hepatotoxicity & $13(7.7 \%)$ & $11(21.6 \%)$ & $24(10.9 \%)$ \\
\hline Pancreatitis & $0(0 \%)$ & $6(11.8 \%)$ & $6(2.7 \%)$ \\
\hline Dyspepsia & $3(1.8 \%)$ & $4(7.8 \%)$ & $7(3.2 \%)$ \\
\hline Allergic reaction & $0(0 \%)$ & $2(3.9 \%)$ & $2(0.9 \%)$ \\
\hline Other & $0(0 \%)$ & $4(7.8 \%)$ & $4(1.8 \%)$ \\
\hline No side effect & $137(81.1 \%)$ & $8(15.7 \%)$ & $145(65.9 \%)$ \\
\hline
\end{tabular}

mutation were grouped together and analyzed as one group. Adverse drug reactions occurred in 13/15 (86.7\%) of patients with any TMPT $* 3$ mutation as compared to $62 / 205(30.2 \%)$ of patient with wild type TPMT genotype, $\mathrm{p}=2.10^{-5}$, OR 15.0 (95\% CI $3.3-$ 68.5) (Tab. 3). There was a striking difference as to type of adverse drug reactions experienced. Bone marrow suppression of various severity occurred in 11/15 (73.3 \%) of patients with TPMT mutations as compared to only $21 / 205(10.2 \%)$ of patients with TPMT wild type genotype, $\mathrm{p}=1.10^{-6}$, OR 24.1 (95\% CI 7.0-82.4). The occurrence of more severe bone marrow suppression (bi- or pancytopenia) was non-significantly more prevalent in patients with TPMT $* 3$ mutation $3 / 11(27.3 \%)$ versus $3 / 21(14.2 \%), p=0.37$.
There was however no difference in the prevalence of other than hematologic adverse drug reactions between the two groups. Gastrointestinal adverse drug reactions occurred in 1/15 (6.7\%) of patients with TPMT mutation versus 36/205 (17.6\%) of patients with wild type TPMT genotype, $\mathrm{p}=0.18$.

There was one patient homozygous for TPMT *3A genotype. In 1997, a rectovesical fistula was diagnosed and azathioprine 50 mg was added to 5-ASA and corticosteroid regime. Two weeks after the onset of AZA therapy a severe pancytopenia developed with WBC $2.4 \times 10^{9} / \mathrm{L}, \mathrm{Hb} 97 \mathrm{~g} / \mathrm{L}$ and platelets $147 \times 10^{9} / \mathrm{L}$. Azathioprine was stopped immediately and the pancytopenia resolved spontaneously within four weeks.

Clinical circumstances of adverse drug reactions to AZA with respect to TPMT genotype

Next we analyzed the difference among patients with TPMT wild type and $* 3$ mutations with respect to the onset, AZA dose and severity of ADR. We analyzed the data separately for hematologic and non-hematologic ADRs (Tab. 4). In the hematologic ADR subgroup we observed a more severe myelotoxicity in a subgroup of patients with the TPMT mutations (mean WBC $2.0 \pm 0.8 .10^{9} / \mathrm{L}$ and hemoglobin $87.7 \pm 12.1 \mathrm{~g} / \mathrm{L}$ ) as compared with the patients with wild type TPMT (mean WBC $2.5 \pm 0.6 .10^{9} / \mathrm{L}$; $\mathrm{p}=0.007, \mathrm{Hb} 116.0 \pm 6.5 \mathrm{~g} / \mathrm{L}, \mathrm{p}=0.03)$. There were no significant differences observed in the non-hematologic adverse drug reactions subgroup.

Tab. 3. Prevalence of adverse drug reactions in our cohort according to the TPMT genotype.

\begin{tabular}{|c|c|c|c|c|}
\hline \multirow[t]{2}{*}{ Adverse drug reaction type } & \multirow{2}{*}{$\begin{array}{l}\text { Adverse drug reactions } \\
\qquad(\mathrm{n}=220)\end{array}$} & \multicolumn{2}{|c|}{ TPMT genotype } & \multirow[t]{2}{*}{$\mathrm{p}$ value } \\
\hline & & Wild type $(n=205)$ & Mutation $(n=15)$ & \\
\hline Any ADR & $75(34.1 \%)$ & $62(30.2 \%)$ & $13(86.7 \%)$ & 0.00002 \\
\hline Myelotoxicity & $32(14.5 \%)$ & $21(10.2 \%)$ & $11(73.3 \%)$ & 0.000001 \\
\hline Leukopenia & $26(11.8 \%)$ & $18(8.8 \%)$ & $8(53.3 \%)$ & \\
\hline Bi- or Pancytopenia & $6(2.7 \%)$ & $3(1.5 \%)$ & $3(20.0 \%)$ & \\
\hline Gastrointestinal ADR & $37(16.8 \%)$ & $36(17.6 \%)$ & $1(6.7 \%)$ & 0.18 \\
\hline Hepatotoxicity & $24(10.9 \%)$ & $23(11.2 \%)$ & $1(6.7 \%)$ & n.s. \\
\hline Dyspepsia & $7(3.2 \%)$ & $7(3.4 \%)$ & $0(0 \%)$ & n.s. \\
\hline Allergic reaction & $2(0.9 \%)$ & $2(1.0 \%)$ & $0(0 \%)$ & n.s. \\
\hline Other & $4(1.8 \%)$ & $3(1.5 \%)$ & $1(6.7 \%)$ & n.s. \\
\hline
\end{tabular}

Tab. 4. Studied clinical and laboratory parameters at the time of ADR in patients with myelotoxicity after AZA therapy.

\begin{tabular}{|c|c|c|c|c|c|c|c|c|c|}
\hline \multirow[t]{3}{*}{ Characteristics } & \multicolumn{8}{|c|}{ TPMT genotype } & \multirow{3}{*}{$\begin{array}{c}\mathrm{p} \\
\text { value }\end{array}$} \\
\hline & \multicolumn{4}{|c|}{ Wild type } & \multicolumn{4}{|c|}{ Mutation } & \\
\hline & $\mathrm{N}$ & Mean & SD & $\begin{array}{l}\text { SE of } \\
\text { Mean }\end{array}$ & $\mathrm{N}$ & Mean & SD & $\begin{array}{l}\text { SE of } \\
\text { Mean }\end{array}$ & \\
\hline ADR onset after therapy initiation (months) & 20 & 15.5 & 14.7 & 3.3 & 11 & 10.5 & 10.6 & 3.2 & n.s. \\
\hline AZA dose at the time of ADR(mg) & 19 & 125.0 & 34.4 & 7.9 & 10 & 110.0 & 39.4 & 12.5 & n.s. \\
\hline AZA dose at the time of ADR $(\mathrm{mg} / \mathrm{kg})$ & 19 & 2.02 & 0.46 & 0.11 & 10 & 1.73 & 0.74 & 0.24 & 0.19 \\
\hline \multicolumn{10}{|l|}{ Laboratory results at the time of ADR } \\
\hline WBC (109/L) & 17 & 2.5 & 0.6 & 0.2 & 9 & 2.0 & 0.8 & 0.3 & 0.007 \\
\hline Lymphocytes(109/L) & 13 & 0.7 & 0.3 & 0.1 & 7 & 1.1 & 1.0 & 0.4 & n.s. \\
\hline Hemoglobin $(g / 1)$ & 17 & 116.0 & 26.8 & 6.5 & 8 & 87.7 & 34.2 & 12.1 & 0.03 \\
\hline $\operatorname{MCV}(f l)$ & 14 & 91.4 & 27.6 & 7.4 & 7 & 93.8 & 8.6 & 3.3 & n.s. \\
\hline Platelets (109/L) & 17 & 253.9 & 94.1 & 22.8 & 8 & 216.9 & 115.0 & 40.6 & n.s. \\
\hline Creatine $(\mu \mathrm{mol} / \mathrm{L})$ & 16 & 65.8 & 16.4 & 4.1 & 8 & 63.9 & 18.3 & 6.5 & n.s. \\
\hline
\end{tabular}

$\mathrm{ADR}$ - adverse drug reaction, $\mathrm{SD}$ - standard deviation, $\mathrm{SE}$ - standard error 
When we analyzed the data on the time to onset of adverse drug reactions separately for hematologic and non-hematologic ADRs and TPMT genotype, we found no significant difference in either subgroup. Myelotoxicity occurred within the first year of therapy in all but 2 patients in the TPMT mutation subgroup (Fig. 2). In the non-hematologic side effects subgroup, adverse drug reactions occurred in $25 / 30$ patients within the first three months of therapy.

\section{The effect of mesalazine on the occurrence of side effects}

All patients used mesalazine as 5-ASA medication. Data on concomitant use of mesalazine at the time of adverse drug reaction were available in 69/75 patients. Concomitant mesalazine use was recorded in 51/69 (73.9\%) patients at the time of adverse drug reaction. Concomitant mesalazine use among patients currently on azathioprine (and no ADRs) was 112/169 (66.3\%), p=n.s. There was also no difference in the presence of particular side effect, severity of bone marrow suppression, and studied laboratory parameters among concomitant mesalazine users and non-users at the time of ADR

\section{Discussion}

In our retrospective multicenter study we analyzed the impact of TMPT genotype on the incidence and characteristics of adverse drug reactions in a Slovak cohort of 220 IBD patients treated with azathioprine.

\section{Limitations of the study}

There are two sources of potential bias in our study that we are aware of. Firstly, all participating centers are tertiary referral IBD centers. There are many patients referred to these centers for the application of biological therapy, including those with AZA intolerance. This presents a potential selection bias which can therefore expectedly increase the incidence of adverse reactions in our cohort compared to general IBD population. On the other hand, we were able to analyze a larger subgroup of patients with ADRs in our cohort. We do not expect the potential referral bias to have other effect than that of increased incidence of ADRs in our cohort, thus the proportion of particular ADRs and the associations with TPMT genotype are not affected.

The retrospective design of our study is the second general limitation. The recall and retrieval bias potentially limits especially the incidence of mild adverse drug reactions (e.g. mild nausea) and studied circumstances of AZA adverse drug reactions such as exact time to ADR, concomitant medication and laboratory results at the time of ADR etc. We tried to overcome this bias by careful inspection of physical as well as electronic patient data files including laboratory results. In case of referred patients (approximately $30 \%$ of the cohort), the referring physician was contacted and consulted if necessary.

\section{Adverse drug reactions}

In our study we observed a high incidence of ADRs to AZA therapy. Myelotoxicity was observed in $14.5 \%$ of our patients, hepatotoxicity in $10.9 \%$, dyspepsia in $3.2 \%$, pancreatitis in $2.7 \%$, allergic reactions in $0.9 \%$ and other in $1.8 \%$. Generally the reported total AZA toxicity in published studies is in a range of $10-20 \%(19,20)$ depending on the cohort of patients. Some authors however report up to $25 \%$ frequency of myelotoxicity in AZA-treated patients (21). We think however that the reason for the observed high frequency of ADRs in our cohort is the above discussed referral character of the participating IBD centers. Furthermore, in the participating IBD centers, the patients are meticulously monitored for ADRs after the initiation of AZA therapy including regular biochemistry monitoring (hepatic and pancreatic enzymes). This might also in part explain the relatively high frequency of hepatotoxicity in our cohort.

The problem of gastrointestinal ADRs (except for pancreatitis) can usually be overcome through switching from AZA to 6-MP or in selected cases to thioguanine (22). Myelotoxicity is more difficult to address. It can be attempted to discontinue the drug administration and/or reduce the dose, however it is a risky approach and in many cases, thiopurines must be stopped.

\section{Prevalence of TPMP}

The frequency of patients with TPMT $* 3$ mutation in our cohort was $6.8 \%$ including $13 / 220(6 \%)$ TPMT $* 3$ A heterozygote patients, $1 / 220(0.4 \%)$ TPMT $* 3 \mathrm{C}$ heterozygote, and 1/220 (0.4 $\%)$ TPMT $* 3 \mathrm{~A}$ homozygote. That corresponds to allele frequencies of $3.4 \%$ for TPMT $* 3 \mathrm{~A}$ allele and $0.2 \%$ for the TPMT $* 3 \mathrm{C}$ allele. This is in accordance with the allele frequencies found in most studies in Caucasians. The allele frequencies of common TPMT mutations in central and eastern European countries are in a range of 3.2-4.9\% (23-27). In a Czech study on 398 IBD patients and 300 healthy controls, the frequencies of TPMT $* 2$, TPMT*3A, TPMT*3B, and TPMT*3C were $0.1 \%, 4.3 \%, 0.1 \%$, and $0.4 \%$, respectively (23). In a Polish study on 358 healthy subjects, the allelic frequencies were $0.4 \%$ for TPMT*2, $2.7 \%$ for TPMT*3A, and $0.1 \%$ for TPMT*3C, respectively. The TPMT*3B allele was not found in the studied population (24).

\section{Association of TPMT and adverse drug reactions}

In our study we observed a strong association between TPMT polymorphisms and hematologic adverse drug reactions. There was however no association with gastrointestinal or any other adverse drug reactions. Bone marrow suppression occurred in $73 \%$ of patients with variant-type TPMT and only in $10 \%$ of TPMT wild-type patients $\left(\mathrm{p}=1.10^{-6}\right)$. There is no doubt about the association of homozygote TPMT *3A genotype with severe bone marrow suppression (28). However the association of heterozygotes with intermediate TPMT activity and myelotoxicity was not clear-cut. There were several studies that suggested no significant association between TPMT and myelotoxicity in heterozygotes $(15,29,30)$. Colombel et al studied a cohort of 41 Crohn's disease patients with leukopenia or thrombocytopenia during AZA or 6-MP therapy, in which $73 \%$ carried high methylator genotype of TPMT, $10 \%$ were homozygous for low-methylator genotype and $17 \%$ were heterozygous for intermediate-methylator genotype of TPMT (29). In another study by Dubinsky et al there were 8 out of $92(90 \%)$ pediatric patients heterozygous for TPMT *3 allele (15). Only one TPMT variant- 
type patient developed leukopenia as compared to 35 of 84 normal-type patients. On the other hand there are some well designed and well powered studies showing a strong association of TPMT heterozygotes with leukopenia. Zelinkova et al studied a cohort of 262 IBD patients (67 UC and $195 \mathrm{CD}$ patients) treated with AZA and genotyped for ITPA and TPMT genotypes (31). Four out of 24 (16.7\%) patients with TPMT mutation developed leukopenia as compared to 8 out of $238(3.4 \%)$ TPMT wild-type patients; $p=0.02$.

Recently there were two meta-analyses of TPMT status and hematologic toxicity published. Dong et al published a meta-analysis of nine studies performed in IBD patients (32). Five of the studies in the meta-analysis showed an association between TPMT mutations and myelotoxicity while four failed to demonstrate this association. The pooled results showed a significant association between TPMT genotype and myelotoxicity. In the pooled data 23/110 (20.9\%), patients with TPMT variant genotype but only $77 / 1309$ (5.9\%) patients with wild-type genotype experienced bone marrow toxicity giving a pooled $\mathrm{OR}=5.93$ (95\% CI $2.96-$ $11.88), \mathrm{p}=0.00001$. No association was confirmed for hepatotoxicity or pancreatitis. The frequency of myelotoxicity among TPMT heterozygotes in analysed studies ranged from $0-60 \%$. In another more specific meta-analysis by Higgs et al, only patients with intermediate TPMT activity were compared to wild-type TPMT patients (28). The study was not limited to certain disease and included 67 mostly retrospective studies. The odds ratio for developing leukopenia for patients with intermediate TPMT activity compared to the normal TPMT patients was 4.19 (95\% CI: 3.20-5.48).

\section{TPMT and dose, time to AE, severity, 5-ASA}

In our study the patients with TPMT $* 3$ mutation developed a significantly more severe bone marrow suppression (WBC $2.0 \pm 0.8 .10^{9} / \mathrm{L}$ ) as compared with the patients with wild type TPMT (WBC $2.5 \pm 0.6 .10^{9} / \mathrm{L} ; \mathrm{p}=0.007$ ). There are few studies, which address the issue of adjusting AZA dose in patients with TPMP mutation. In a study with 71 patients by Regueiro et al, the incidence of ADRs was recorded after starting AZA over a period of one year including 8 patients with intermediate TPMT activity (33). Seven patients were started on 1 to $1.5 \mathrm{mg} / \mathrm{kg} / \mathrm{d}$ ofAZA, and one did not receive treatment. None of the TPMT intermediate patients receiving a reduced dose

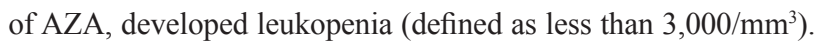

In our study we have not observed any clinically significant interaction between AZA and 5-ASA in terms of the incidence of ADRs. Earlier studies suggested an inhibitory effect of sulfasalazine and TPMT (34). Newer studies suggest no significant ex vivo effect of now widely used mesalazine (35). Gilissen et al studied a small cohort of 17 patients on concomitant 6-MP $(0.78 \mathrm{mg} / \mathrm{kg} /$ day $)$ and mesalazine (3g/day) therapy (36). After mesalazine discontinuation, mean tioguanine levels changed significantly from 262 to $209 \mathrm{pmol} / 8 \times 10^{8} \mathrm{red}$ blood cells, increasing to 270 after reintroduction. Leucocyte counts however were stable. In a clinical study on 186 IBD patients, concurrent use of 5-aminosalicylate drugs did not reduce the relapse rates of inflammatory bowel disease in patients established on azathioprine therapy (37). Furthermore the use of 5-aminosalicylate drugs did not lead to any increase in discontinuation of azathioprine due to adverse drug reactions.
There is an ongoing discussion, whether to perform TPMT genotyping and/or measurement of TPMT activity before initiating an AZA therapy in IBD patients. The American Gastroenterological Association issued a medical position statement on TPMT genotyping while reflecting the current FDA recommendation that in an effort to detect individuals with low enzyme activity, The patients should have their TPMT genotype or phenotype assessed before the initiation of AZA therapy (4). The European Crohn's and Colitis Organisation (ECCO) recommends not to yet consider TPMT status determination as a pre-requisite to AZA or 6-MP therapy (5).

Our results suggest that the myelotoxocity could be prevented in around $5 \%$ of our patients $(73 \%$ positive predictive value in $6.8 \%$ TPMT variant patients of the cohort). This includes patients developing more severe bi- or pancytopenia representing $1.4 \%$ of genotyped patients. Considering the more conservative $20 \%$ positive predictive value of TPMT heterozygous status for myelotoxicity observed in meta-analysis by Dong et al, it could still be prevented in about $1.5 \%$ of patients (32). We believe this is an argument for TPMT genotyping or activity measurement in all IBD patients considered for AZA therapy. If the TPMT status determination is not routinely performed, it should be strongly considered at least in patients in whom bone marrow suppression can be potentially devastating such as in elderly, patients with known severe medical condition or patients with moderate to severely impaired renal function.

\section{Conclusion}

The prevalence of TPMT mutation in the Slovak population is comparable to other studies in Caucasians and led to bone marrow suppression in a significant proportion of our patients. TPMT genotyping could have prevented myelotoxicity in about $5 \%$ of our patients. TPMT genotyping of activity measurement should be performed at least in high-risk subgroups of IBD patients in whom AZA therapy is considered.

\section{References}

1. Pearson DC, May GR, Fick GH, Sutherland LR. Azathioprine and 6-mercaptopurine in Crohn disease. A meta-analysis. Ann Intern Med 1995; 123: $132-142$.

2. Adler DJ, Korelitz BI. The therapeutic efficacy of 6-mercaptopurine in refractory ulcerative colitis. Am J Gastroenterol 1990; 85: 717-722.

3. Jewell DP, Truelove SC. Azathioprine in ulcerative colitis: an interim report on a controlled therapeutic trial. Br Med J 1972; 1: 709-712.

4. Lichtenstein GR, Abreu MT, Cohen R, Tremaine W. American Gastroenterological Association Institute medical position statement on corticosteroids, immunomodulators, and infliximab in inflammatory bowel disease. Gastroenterology 2006; 130: 935-939.

5. Travis SP, Stange EF, Lemann M, Oresland T, Chowers Y, Forbes A et al. European evidence based consensus on the diagnosis and management of Crohn's disease: current management. Gut 2006; 55 Suppl 1: i16-35.

6. Travis S, Stange E, Lemann M, Oresland T, Bemelman WA, Chowers $\mathbf{Y}$ et al. European evidence-based consensus on the management of ulcerative colitis: Current management. Journal of Crohn's and Colitis 2008; 2: 24-62. 
7. Bouhnik Y, Lemann M, Mary JY, Scemama G, Tai R, Matuchansky C et al. Long-term follow-up of patients with Crohn's disease treated with azathioprine or 6-mercaptopurine. Lancet 1996; 347: 215-219.

8. Ardizzone S, Bollani S, Manzionna G, Imbesi V, Colombo E, Bianchi-Porro G. Comparison between methotrexate and azathioprine in the treatment of chronic active Crohn's disease: a randomised, investigatorblind study. 2003; 35: 619-627.

9. Connell WR, Kamm MA, Ritchie JK, Lennard-Jones JE. Bone marrow toxicity caused by azathioprine in inflammatory bowel disease: 27 years of experience. Gut 1993; 34: 1081-1085.

10. Krynetskaia NF, Krynetski EY, Evans WE. Human RNase H-mediated RNA cleavage from DNA-RNA duplexes is inhibited by 6-deoxythioguanosine incorporation into DNA. Mol Pharmacol 1999; 56: 841-848.

11. Tiede I, Fritz G, Strand S, Poppe D, Dvorsky R, Strand D et al. CD28-dependent Rac1 activation is the molecular target of azathioprine in primary human CD4+ T lymphocytes. J Clin Invest 2003; 111: 1133-1145.

12. Thomas CW, Myhre GM, Tschumper R, Sreekumar R, Jelinek D, McKean DJ et al. Selective inhibition of inflammatory gene expression in activated T lymphocytes: a mechanism of immune suppression by thiopurines. J Pharmacol Exp Ther 2005; 312: 537-545.

13. Coulthard SA, Hogarth LA, Little M, Matheson EC, Redfern CP, Minto $\mathbf{L}$ et al. The effect of thiopurine methyltransferase expression on sensitivity to thiopurine drugs. Mol Pharmacol 2002; 62: 102-109.

14. Hindorf U, Peterson C, Almer S. Assessment of thiopurine methyltransferase and metabolite formation during thiopurine therapy: results from a large Swedish patient population. Ther Drug Monit 2004; 26: 673-678.

15. Dubinsky MC, Lamothe S, Yang HY, Targan SR, Sinnett D, Theoret Y et al. Pharmacogenomics and metabolite measurement for 6-mercaptopurine therapy in inflammatory bowel disease. Gastroenterology 2000; 118: 705-713.

16. Dubinsky MC, Yang H, Hassard PV, Seidman EG, Kam LY, Abreu MT et al. 6-MP metabolite profiles provide a biochemical explanation for 6-MP resistance in patients with inflammatory bowel disease. Gastroenterology 2002; 122: 904-915.

17. Lennard L, Van Loon JA, Weinshilboum RM. Pharmacogenetics of acute azathioprine toxicity: relationship to thiopurine methyltransferase genetic polymorphism. Clin Pharmacol Ther 1989; 46: 149-154.

18. Schaeffeler E, Fischer C, Brockmeier D, Wernet D, Moerike K, Eichelbaum $\mathrm{M}$ et al. Comprehensive analysis of thiopurine S-methyltransferase phenotype-genotype correlation in a large population of GermanCaucasians and identification of novel TPMT variants. Pharmacogenetics 2004; 14: 407-417.

19. Ardizzone S, Bollani S, Manzionna G, Imbesi V, Colombo E, Bianchi Porro G. Comparison between methotrexate and azathioprine in the treatment of chronic active Crohn's disease: a randomised, investigatorblind study. Dig Liver Dis 2003; 35: 619-627.

20. Nielsen OH, Vainer B, Rask-Madsen J. Review article: the treatment of inflammatory bowel disease with 6-mercaptopurine or azathioprine. Aliment Pharmacol Ther 2001; 15: 1699-1708.

21. Panaccione R, Fedorak R. Primer of current therapies to treat inflammatory bowel disease - Purine analogs. In: Satsangi J, Sutherland L, Colombel JF, Fiocchi C, Lofberg R, Pemberton JH et al (Eds). Inflammatory bowel diseases: Churchill Livingstone; 2003; p. 343-46.

22. Bonaz B, Boitard J, Marteau P, Lemann M, Coffin B, Flourie B et al. Tioguanine in patients with Crohn's disease intolerant or resistant to azathioprine/mercaptopurine. Aliment Pharmacol Ther 2003; 18: 401-408.
23. Slanar O, Bortlik M, Buzkova H, Donoval R, Pechandova K, Sebesta I et al. Polymorphisms of the TPMT gene in the Czech healthy population and patients with inflammatory bowel disease. Nucleosides Nucleotides Nucleic Acids 2008; 27: 835-838.

24. Kurzawski M, Gawronska-Szklarz B, Drozdzik M. Frequency distribution of thiopurine S-methyltransferase alleles in a polish population. Ther Drug Monit 2004; 26: 541-545.

25. Samochatova EV, Chupova NV, Rudneva A, Makarova O, Nasedkina TV, Fedorova OE et al. TPMT genetic variations in populations of the Russian Federation. Pediatr Blood Cancer 2009; 52: 203-208.

26. Dokmanovic L, Urosevic J, Janic D, Jovanovic N, Petrucev B, Tosic $\mathbf{N}$ et al. Analysis of thiopurine S-methyltransferase polymorphism in the population of Serbia and Montenegro and mercaptopurine therapy tolerance in childhood acute lymphoblastic leukemia. Ther Drug Monit 2006; 28: 800-806.

27. Milek M, Murn J, Jaksic Z, Lukac Bajalo J, Jazbec J, Mlinaric Rascan I. Thiopurine S-methyltransferase pharmacogenetics: genotype to phenotype correlation in the Slovenian population. Pharmacology 2006; 77: $105-114$.

28. Higgs JE, Payne K, Roberts C, Newman WG. Are patients with intermediate TPMT activity at increased risk of myelosuppression when taking thiopurine medications? Pharmacogenomics 2010; 11: 177-188.

29. Colombel JF, Ferrari N, Debuysere H, Marteau P, Gendre JP, Bonaz B et al. Genotypic analysis of thiopurine S-methyltransferase in patients with Crohn's disease and severe myelosuppression during azathioprine therapy. Gastroenterology 2000; $118: 1025-1030$.

30. Gearry RB, Barclay ML, Burt MJ, Collett JA, Chapman BA, Roberts RL et al. Thiopurine S-methyltransferase (TPMT) genotype does not predict adverse drug reactions to thiopurine drugs in patients with inflammatory bowel disease. Aliment Pharmacol Ther 2003; 18: 395-400.

31. Zelinkova Z, Derijks LJ, Stokkers PC, Vogels EW, van Kampen AH, Curvers WL et al. Inosine triphosphate pyrophosphatase and thiopurine s-methyltransferase genotypes relationship to azathioprine-induced myelosuppression. Clin Gastroenterol Hepatol 2006; 4: 44-49.

32. Dong XW, Zheng Q, Zhu MM, Tong JL, Ran ZH. Thiopurine Smethyltransferase polymorphisms and thiopurine toxicity in treatment of inflammatory bowel disease. World J Gastroenterol 2010; 16: 3187-3195.

33. Regueiro M, Mardini H. Determination of thiopurine methyltransferase genotype or phenotype optimizes initial dosing of azathioprine for the treatment of Crohn's disease. J Clin Gastroenterol 2002; 35: 240-244.

34. Szumlanski CL, Weinshilboum RM. Sulphasalazine inhibition of thiopurine methyltransferase: possible mechanism for interaction with 6-mercaptopurine and azathioprine. Br J Clin Pharmacol 1995; 39: 456-459.

35. Xin HW, Fischer C, Schwab M, Klotz U. Thiopurine S-methyltransferase as a target for drug interactions. Eur J Clin Pharmacol 2005; 61: 395-398.

36. Gilissen LP, Bierau J, Derijks LJ, Bos LP, Hooymans PM, van Gennip A et al. The pharmacokinetic effect of discontinuation of mesalazine on mercaptopurine metabolite levels in inflammatory bowel disease patients. Aliment Pharmacol Ther 2005; 22: 605-611.

37. Campbell S, Ghosh S. Effective maintenance of inflammatory bowel disease remission by azathioprine does not require concurrent 5 -aminosalicylate therapy. Eur J Gastroenterol Hepatol 2001; 13: 1297-1301.

Received February 24, 2011. Accepted October 20, 2011. 